\title{
Effects of curcumin on global gene expression profiles in the highly invasive human breast carcinoma cell line MDA-MB 231: A gene network-based microarray analysis
}

\author{
NACI CINE $^{1 *}$, PORNNGARM LIMTRAKUL $^{2 *}$, DENIZ SUNNETCI $^{1}$, BALINT NAGY $^{3}$ and HAKAN SAVLI ${ }^{1}$ \\ ${ }^{1}$ Department of Medical Genetics and Clinical Research Unit, Faculty of Medicine, Kocaeli University, Kocaeli, Turkey; \\ ${ }^{2}$ Biochemistry Department, Chiang Mai University, Thailand; ${ }^{3}$ First Department of Obstetrics and Gynecology, \\ Genetics Laboratory, Semmelweis University, Budapest, Hungary
}

Received July 30, 2012; Accepted October 9, 2012

DOI: 10.3892/etm.2012.754

\begin{abstract}
Curcumin, or diferuloylmethane, is a major chemical component of turmeric (Curcuma longa Linn.) that has been consumed as a dietary spice through the ages. This yellow-colored polyphenol has a notably wide range of beneficial properties, including anti-inflammatory, antioxidant, antitumoral, anti-invasive and anti-metastatic activity. In the present study, microarray gene expression analysis was applied to identify the curcumin-regulated genes in a highly invasive human breast carcinoma cell line (MDA-MB 231). Cells were cultured with curcumin $(20 \mu \mathrm{M})$ for $24 \mathrm{~h}$; total RNA was isolated and hybridized to Whole Human Genome Microarray slides. Gene set enrichment analyses on our whole genome expression data revealed downregulation of the EGF pathway elements following curcumin treatment. Furthermore, gene network analysis identified a significantly relevant network among the differentially expressed genes, centered on the EGRI and FOS genes. The members of these pathways and networks play an essential role in the regulation of cancer cell growth and development; the majority exhibited decreased expression levels following treatment with curcumin. These observations suggest that curcumin is an excellent candidate for the prevention and treatment of breast cancer.
\end{abstract}

\section{Introduction}

The polyphenol curcumin is the active ingredient in the herbal remedy and dietary spice turmeric (Curcuma longa Linn.). Turmeric has been widely used in traditional Indian medicine

Correspondence to: Professor Pornngarm Limtrakul, Biochemistry Department, Chiang Mai University, 239 Huay Kaew Road, Muang District, Chiang Mai, Thailand, 50200

E-mail: plimtrak@med.cmu.ac.th

*Contributed equally

Key words: curcumin, microarray, gene expression, breast cancer to cure biliary disorders, anorexia, coughs, diabetic wounds, hepatic disorders, rheumatism and sinusitis since the time of Ayurveda (1). Extensive research over the last decade has revealed that this molecule is capable of reducing blood cholesterol (2), preventing low-density lipoprotein oxidation (3) and inhibiting platelet aggregation (1). Curcumin is capable of exerting a wide range of antiproliferative and proapoptotic effects against various tumors in vitro (4-6) and in vivo. The compound has been identified as being able to successfully prevent carcinogenesis of the breast (7) and various other organs (8-10), targeting multiple steps in the pathway to malignancy. Additionally, curcumin has been shown to suppress thrombosis (11) and myocardial infarction (12) and to suppress symptoms associated with type II diabetes (13) and rheumatoid arthritis (14). These pleiotropic molecular effects may be mediated by the inhibitory effect curcumin has on a wide variety of cell signaling factors, including AP-1 transcription factor, c-Myc, EGR-1, NF- $\kappa \mathrm{B}$, protein kinase $\mathrm{C}$, epidermal growth factor receptor tyrosine kinase, c-Jun $\mathrm{N}$-terminal kinase, protein tyrosine kinases, protein serine/threonine kinases and IкB kinase (15-17).

Metastasis is a complex integrated process that involves interactions between cancer cells and their surrounding microenvironments, and it is one of the most significant deciding factors in the outcome of cancer. The majority of drugs currently available for the treatment of cancer have limited potential as they are extremely toxic, highly inefficient in treating cancer or highly expensive. Therefore, treatments without these disadvantages are certainly required. Curcumin is one such agent; several studies have shown that curcumin is able to kill cancer cells selectively (18-20). Curcumin has previously been identified to exhibit antimetastatic activity in in vivo animal models $(21,22)$. Oral administration of curcumin was revealed to significantly inhibit lung metastasis (21), and curcumin treatment significantly inhibited the invasion of B16F-10 melanoma cells (22). These results indicate a possible use of curcumin as an antimetastatic agent, but the mechanism involved behind these beneficial effects of the ideal 'Spice for Life' remains unknown.

The advantages of high-throughput microarray technologies offer a new opportunity to gain insight into the global gene 
expression changes induced by curcumin in various highly metastatic carcinoma cell lines, leading to the identification of new curcumin-regulated genes and pathways. Previously, researchers observed an anti-invasive gene expression profile following curcumin treatment in lung adenocarcinoma, based on a cDNA microarray analysis (23). These studies also highlighted that several additional, and as of yet unidentified, gene interactions may be responsible for the multiple beneficial effects of curcumin. The aim of our current study was to identify novel curcumin-regulated genes, gene networks and pathways in a highly invasive human breast carcinoma cell line (MDA-MB 231) by applying microarray gene expression analysis subsequent to $24 \mathrm{~h}$ of curcumin treatment. Furthermore, to enable the integration of our results into multiple levels of information available in public databases, we applied gene set enrichment analysis (GSEA) and gene network analysis to our microarray data.

\section{Materials and methods}

Materials. Dulbecco's modified Eagle's medium (DMEM), penicillin-streptomycin and trypsin-EDTA were purchased from Gibco-BRL (Grand Island, NY, USA). Fetal calf serum (FCS) was purchased from Hyclone (Logan, UT, USA). MTT (3-(4,5-dimethylthiazol-2-yl)-2,5-diphenyltetrazolium bromide) was purchased from Sigma (St. Louis, MO, USA).

Cell line and culture conditions. The MDA-MB 231 human invasive breast carcinoma cell line was purchased from the American Type Culture Collection (ATCC; Manassas, VA, USA). The cells were grown in Leibovitz L-15 medium supplemented with $100 \mathrm{U} / \mathrm{ml}$ penicillin, $100 \mathrm{mg} / \mathrm{ml}$ streptomycin and $10 \%$ heat-inactivated FCS. The cultures were maintained at $37^{\circ} \mathrm{C}$ in a humidified atmosphere without $\mathrm{CO}_{2}$.

Extraction and isolation of curcuminoids. Curcumin was isolated and purified as previously described (24). Briefly, Chiang Mai turmeric powder (1 kg) was successively extracted with hexane (2.51) and 95\% ethanol (7.51) at room temperature. Turmeric curcuminoids were then precipitated with petrolium ether yielding $50 \mathrm{~g}$ crude curcuminoid mixtures $(78 \%$ curcumin, $16 \%$ demethoxycurcumin and 5\% bisdemethoxycurcumin). The curcuminoids ( $3 \mathrm{~g}$ ) were further fractionated by Silica gel 60 column chromatography $(44 \times 1.6 \mathrm{~cm})$ using first $\mathrm{CHCl}_{3}$ and then $\mathrm{CHCl}_{3} / \mathrm{MeOH}$ with increasing polarity. Fractions containing curcumin $(1.11 \mathrm{~g})$ were eluted with $100 \%$ $\mathrm{CHCl}_{3}$ (0.6 1). Fractions containing demethoxycurcumin (200 $\mathrm{mg})$ and bisdemethoxycurcumin (40 mg) were further eluted with $\mathrm{CHCl}_{3} / \mathrm{MeOH}\left(98: 2,0.8\right.$ 1) and $\mathrm{CHCl}_{3} / \mathrm{MeOH}$ (95:5, 11 ), respectively.

MTT assay of cell viability. Cell viability was measured using the conventional MTT reduction assay as described previously (25). Briefly, MDA-MB 231 cells were inoculated at a density of $5 \times 10^{3}$ cells/well in 96-well plates for $24 \mathrm{~h}$, in $200 \mu \mathrm{l}$ DMEM with $10 \%$ FCS. The culture supernatant was removed and DMEM with $10 \%$ FCS containing various concentrations of curcumin was added and incubated for $24 \mathrm{~h}$. MTT dye $(10 \mu 1,5 \mathrm{mg} / \mathrm{ml})$ was added and the plate was incubated for an additional $4 \mathrm{~h}$. The absorbance of MTT-formazan was measured using a microplate reader at $540 \mathrm{~nm}$ with a reference wavelength of $630 \mathrm{~nm}$.

Preparation of cell pellets. MDA-MB 231 cells were seeded into a $75 \mathrm{~mm}^{3} \mathrm{~T}$ flask in DMEM with $10 \%$ FCS. Subconfluent cell cultures were incubated for $24 \mathrm{~h}$ in nontoxic concentrations of curcumin $(20 \mu \mathrm{M})$ in DMEM with $10 \%$ FCS. Following treatment, the cells were washed twice with ice-cold PBS and then scraped with a cell scraper into further ice-cold PBS. The cells were centrifuged at $500 \mathrm{x} g$ for $10 \mathrm{~min}$, the supernatant was removed and the cell pellets were lysed.

RNA isolation, quality and quantity determination. Cellular total RNA was prepared by RNeasy columns (Qiagen, Valencia, CA, USA). The quality and quantity of total RNA was determined with an Agilent 2100 Bioanalyzer (Agilent Technologies, Palo Alto, CA, USA). Only those samples that yielded $>8.0$ for RNA integrity number, exhibited a clear gel image and for which no DNA contamination was observed on the histogram, were used for microarray or real-time PCR experiments.

Quantitative real-time PCR ( $Q-R T-P C R)$. cDNA was synthesized using DNase-I-treated total RNA with a First Strand cDNA Synthesis kit for RT-PCR (Roche Diagnostic Corp., Indianapolis, IN, USA) according to the manufacturer's instructions. Q-PCR for determination of FOS, EGRI and GDF15 gene expression was performed using a LightCycler (Roche Diagnostic Corp.) as described previously (26). The G6PD gene was used as an internal control. The gene sequences are shown in Table I.

Microarray measurements, data normalization and analysis. The 1000 ng quality-checked total cellular RNA was reverse transcribed using a Low RNA Input Linear Amplification kit (Agilent Technologies) and then transcribed to Cy3-labeled cRNA according to the manufacturer's instructions. The labeled cRNA was purified (RNeasy kit; Qiagen) and the dye content ( $>9.0 \mathrm{pmol}$ dye $/ \mu \mathrm{g}$ cRNA) and concentration of cRNA was measured using a NanoDrop ND-1000 spectrophotometer (NanoDrop Technologies, Wilmington, DE, USA). Cy3-labeled cRNA (1650 ng) was hybridized to Whole Human Genome Oligo 4x44 k microarrays overnight at $65^{\circ} \mathrm{C}$, then the slides were washed and treated with Stabilizing and Drying Solution (Agilent Technologies) and scanned by an Agilent Microarray Scanner. All steps were carried out according to the manufacturer's instructions. Data were normalized by the Feature Extraction software, version 7.5, with default parameter settings for one-color oligonucleotide microarrays and then transferred to the GeneSpring 9.02 program (Agilent Technologies) for further statistical evaluation. The normalization and data transformation steps recommended by Agilent Technologies for one-color data were applied once in GeneSpring. Experimental interpretation was built by GeneSpring, and the expressed genes that exhibited a $>2.0$-fold differential expression were further analyzed by statistical tests. The statistical comparisons were performed between the groups by performing an unpaired t-test. The multiple testing correction method designed by 
Table I. Fold changes of gene expression by microarray and qRT-PCR relative to the controls.

\begin{tabular}{lcr} 
Genes & $\begin{array}{c}\text { Fold changes obtained by } \\
\text { real-time PCR }\end{array}$ & $\begin{array}{c}\text { Fold changes obtained by } \\
\text { microarray }\end{array}$ \\
\hline FOS & $2,452,436$ (downregulated) & 37,162 (downregulated) \\
EGR1 (Early growth response 1) & 19,027 (downregulated) & 10,685 (downregulated) \\
GDF15 (Growth differentiation factor 15) & 42,518 (downregulated) & 13,484 (downregulated) \\
\hline
\end{tabular}

Table II. Gene set enrichment analysis.

\begin{tabular}{|c|c|c|c|}
\hline Pathway set name & NES & $\begin{array}{c}\text { NOM } \\
\text { P-value }\end{array}$ & $\begin{array}{c}\text { FDR } \\
\text { q-value }\end{array}$ \\
\hline PDGFPATHWAY & 2.1036 & 0.00 & 0.0432097 \\
\hline HSA04520_ADHERENS_JUNCTION & 1.4830 & 0.02 & 0.0433947 \\
\hline EGFPATHWAY & 2.0396 & 0.00 & 0.0455140 \\
\hline CELL_CYCLE_KEGG & 2.0778 & 0.00 & 0.0473429 \\
\hline HSA04640_HEMATOPOIETIC_CELL_LINEAGE & -2.1586 & 0.00 & 0.0192348 \\
\hline ASBCELLPATHWAY & -1.9279 & 0.00 & 0.0338345 \\
\hline
\end{tabular}

A positive normalized enrichment score (NES) indicates correlation with the control group, while negative values indicate correlation with the curcumin-treated group. The nominal (NOM) P-value estimates the statistical significance of the enrichment score for a single gene set. Significant false discovery rate (FDR) and nominal P-values were $<5 \%$ and 0.05 , respectively.

Benjamini-Hochberg was applied with a $\mathrm{P}<0.05$ cut-off value in our statistical tests.

Gene network analysis. For our proposed gene network analysis, gene symbols (Human Gene Organization; HUGO), Agilent probe IDs and the fold change of the significantly differentially regulated genes (curcumin-treated samples versus control) were imported into the IPA 5.0 software (Ingenuity Systems, Redwood City, CA, USA). In IPA, the analysis was carried out with $\mathrm{P}<0.05$ as the cut-off point. Those genes with known gene symbols (HUGO) and their corresponding expression values were uploaded into the software. Each gene symbol was mapped to its corresponding gene object in the Ingenuity Pathways Knowledge Base. Networks of these genes were algorithmically generated based on their connectivity and assigned a score. The score was a numerical value used to rank networks according to their relevance to the genes in the input dataset but this may not have indicated the quality or significance of the network. The score took into account the number of focus genes in the network and the size of the network, to approximate the relevance of this network to the original list of focus genes.

GSEA. The GSEA was carried out using GSEA v2.0 software (27). For the GSEA, a collection of canonical pathway gene sets containing 639 pathways was downloaded from the Molecular Signatures Database (MSigDB) website (http:// www.broadinstitute.org/gsea/msigdb/index.jsp). The pathway gene sets were curated from the Kyoto Encyclopedia of Genes and Genomes (KEGG), BioCarta and GenMAPP online pathway databases. Usually, these gene sets are canonical representations of a biological process compiled by domain experts. Probes were collapsed to HUGO gene names. Gene sets were assessed as to whether they individually scored highly when compared with other possible choices of gene sets. This provided an unbiased means of assessing pathways and of testing gene lists with respect to the enrichment degree of representation of highly regulated genes. A positive normalized enrichment score (NES) indicated correlation with the control, while a negative value indicated correlation with curcumin treatment. The nominal P-value estimated the statistical significance of the enrichment score for a single gene set. We drew conclusions from the top gene sets that had a false discovery rate of $<5 \%$ and a P-value of $<0.05$, both of which are acceptable cut-off values for the identification of biologically relevant gene sets.

\section{Results}

Measurement of cell viability. The number of viable cells following curcumin treatment for $24 \mathrm{~h}$ was estimated using an MTT assay. Exposure of MDA-MB 231 cells to curcumin $(0,10,20,30,40$ and $60 \mu \mathrm{M})$ affected the viability of the cells in a concentration-dependent manner $(96.5 \pm 4.0 \%$ at $10 \mu \mathrm{M}$, $95.2 \pm 5.5 \%$ at $20 \mu \mathrm{M}, 70.3 \pm 3.1 \%$ at $30 \mu \mathrm{M}, 53.3 \pm 4.0 \%$ at $40 \mu \mathrm{M}$ and $24.0 \pm 0.9 \%$ at $60 \mu \mathrm{M}, \mathrm{P}<0.05$, ANOVA) The inhibitory concentration of cell growth at $50 \%$ was $40.0 \pm 2.8 \mu \mathrm{M}$ in the MDA-MB 231 cells $(n=3)$.

Microarray gene expression analysis. Following $24 \mathrm{~h}$ of curcumin treatment ( $20 \mu \mathrm{M} ;>90 \%$ cell survival rate), 35 genes were revealed to be significantly differentially expressed rela- 
tive to the control. This was tested using an Agilent Whole Human Genome (4x44 k) Microarray Platform and traditional microarray data analysis. The list of these genes is shown in Table II.

Verification of selected genes by $Q-R T-P C R$. To substantiate the results of the microarray studies, Q-RT-PCR was performed to assess the mRNA expression of FOS, EGRI and GDF15. As presented in Table I, the microarrays and real-time PCR resulted in similar gene expression changes in the case of the three genes mentioned above, confirming the reliability of our microarray results at the mRNA level.

\section{Discussion}

The anticancer potential of curcumin in various systems has been reviewed previously (28). Curcumin has been shown to block transformation, tumor initiation, tumor promotion, invasion, angiogenesis and metastasis. In the present study, we investigated the effect of 24-h curcumin treatment on the global gene expression profile of the MDA-MB 231 human invasive breast carcinoma cell line. Since the traditional microarray data analysis generated only a moderate number of significantly differentially regulated genes, we applied GSEA on our whole genome gene expression data; this algorithm is designed to effectively evaluate the outcome of a specific experimental condition on known biological pathways and functional categories. In numerous cases, traditional gene expression analyses of large scale microarray data focus on statistically differentially expressed genes, which may lead to a number of potentially significant disease-related genes being bypassed. GSEA is capable of solving this problem by focusing on gene sets rather than individual genes. This method tests the hypothesis of whether members of an a priori defined gene set (e.g., biological pathways) are enriched in the rankordered list. This list is generated from the whole microarray data based on the difference between two biological states (e.g., treated versus control). Our GSEA focused on predefined gene sets from pathway databases. We drew conclusions only from the top gene sets (with a false discovery rate of less than $5 \%$ and a P-value of less than 0.05, Table II). Among these, pathways associated with PDGF, EGF signaling, cell proliferation, the cell cycle and cell junctions exhibited the strongest correlation with the control group, while pathways related to hematopoietic cell lineage and B cells correlated only with the curcumin-treated group. This observation highlights the suppressive effect curcumin has on various cell proliferation pathways by decreasing the expression level of their members. In spite of this, the induction of pathways related to hematopoietic cell lineage and B cells stimulates the activation of several immune function-related cytokines and cell adhesion molecules. These immune-stimulating effects may lead to the activation and attraction of nearby immune cells surrounding the tumor tissue, enhancing the natural defense response of the body against the tumor cells.

These analyses indicate whether a given treatment (e.g., curcumin stimulation) results in enrichment of gene sets that are involved in the regulation of specific pathways (see Materials and methods for details). GSEA clearly revealed that the pathways involved in PTEN, PDGF, EGF signaling, cell proliferation and the cell cycle exhibited significant positive correlation with the control group, while none of these sets were enriched in the curcumin-treated samples, therefore indicating the suppressive effect of curcumin on various cell proliferation pathways. Gene interaction based network investigation of the 35 significantly differently expressed genes obtained by traditional microarray data analysis identified a significantly relevant gene network around FOS and EGRI genes. The members of this network play an essential role in the regulation of cancer cell growth and development; the majority exhibited decreased expression levels following treatment with curcumin.

Overexpression and aberrant function of EGFR have been identified in a variety of human tumors, including colorectal cancer (29). Activation of EGFR is initiated by binding of ligands, including epidermal growth factor (EGF) and transforming growth factor- $\alpha$. This results in the formation of homo- or heterodimers and activation of receptor tyrosine kinase, which, in turn, leads to signaling cascades and regulation of the expression of the target genes. Studies have shown that interruption of EGF signaling impairs tumor growth (30-34). It has previously been demonstrated that curcumin significantly suppressed gene expression of EGRI at transcription and translation levels (35-37). Curcumin successfully reduced the trans-activation activity of the transcription factor EGR-1 by suppressing EGR-1 expression, interrupting the ERK signal pathway and reducing the activity of ELK-1. The authors demonstrated that the reduction of EGR-1 activity plays a critical role in the inhibition of EGFR expression in human colon cancer cells (32). Suppression of EGFR expression and inhibition of receptor tyrosine phosphorylation of EGFR interrupts EGF signaling (38), which collectively contributes to the inhibition of colon cancer cell growth by curcumin. It has been demonstrated that curcumin causes the growth arrest and apoptosis of B-cell lymphoma by downregulation of numerous survival-related genes, including EGRl (39). It must be noted, however, that the role of EGR-1 expression in breast cancer is controversial. In a previous study, EGR-1 expression was revealed to be profoundly reduced in human breast cancer tissues and in various cell lines $(40,41)$. In addition, we have to emphasize that since the in vivo system is multifactorial and more complicated, directly extrapolating in vitro conditions and results into the in vivo system may be misleading. Therefore, further studies are required to validate these results.

In conclusion, by applying various bioinformatical approaches we have revealed the decreased expression of pathway elements in the EGF signaling cascade and the decreased expression of the EGRl gene on the mRNA level in the MDA-MB 231 human invasive breast carcinoma cell line. To the best of our knowledge we are the first to apply comprehensive GSEA and network analysis to analyze the gene expression profile of curcumin treatment on a breast carcinoma cell line. These observations suggest that curcumin may be an excellent candidate for the prevention and treatment of breast cancer.

\section{Acknowledgements}

The authors thank all their colleagues and co-workers from Turkey, Thailand and Hungary for their contributions and support. They also thank the physicians for their participa- 
tion and scientific advice. This study has been generously supported by Kocaeli University BAP (No. 2008/015).

\section{References}

1. Shah BH, Nawaz Z, Pertani SA, Roomi A, Mahmood H, Saeed SA and Gilani AH: Inhibitory effect of curcumin, a food spice from turmeric, on platelet-activating factor- and arachidonic acidmediated platelet aggregation through inhibition of thromboxane formation and $\mathrm{Ca}^{2+}$ signaling. Biochem Pharmacol 58: 1167-1172, 1999.

2. Yuan HY, Kuang SY, Zheng X, et al: Curcumin inhibits cellular cholesterol accumulation by regulating SREBP-1/caveolin-1 signaling pathway in vascular smooth muscle cells. Acta Pharmacol Sin 29: 555-563, 2008

3. Chen WF, Deng SL, Zhou B, Yang L and Liu ZL: Curcumin and its analogues as potent inhibitors of low density lipoprotein oxidation: $\mathrm{H}$-atom abstraction from the phenolic groups and possible involvement of the 4-hydroxy-3-methoxyphenyl groups. Free Radic Biol Med 40: 526-535, 2006.

4. Chen H, Zhang ZS, Zhang YL and Zhou DY: Curcumin inhibits cell proliferation by interfering with the cell cycle and inducing apoptosis in colon carcinoma cells. Anticancer Res 19: 3675-3680, 1999.

5. Kuo ML, Huang TS and Lin JK: Curcumin, an antioxidant and anti-tumor promoter, induces apoptosis in human leukemia cells. Biochim Biophys Acta 1317: 95-100, 1996.

6. Mehta K, Pantazis P, McQueen T and Aggarwal BB Antiproliferative effect of curcumin (diferuloylmethane) against human breast tumor cell lines. Anticancer Drugs 8: 470-481, 1997.

7. Huang MT, Lou YR, Xie JG, Ma W, Lu YP, Yen P, Zhu BT, Newmark $\mathrm{H}$ and Ho CT: Effect of dietary curcumin and dibenzoylmethane on formation of 7,12-dimethylbenz[a] anthracene-induced mammary tumors and lymphomas/leukemias in Sencar mice. Carcinogenesis 19: 1697-1700, 1998.

8. Chuang SE, Kuo ML, Hsu CH, Chen CR, Lin JK, Lai GM, Hsieh CY and Cheng AL: Curcumin-containing diet inhibits diethylnitrosamine-induced murine hepatocarcinogenesis. Carcinogenesis 21: 331-335, 2000.

9. Kawamori T, Lubet R, Steele VE, Kelloff GJ, Kaskey RB, Rao CV and Reddy BS: Chemopreventive effect of curcumin, a naturally occurring anti-inflammatory agent, during the promotion/progression stages of colon cancer. Cancer Res 59: 597-601, 1999.

10. Limtrakul P, Lipigorngoson S, Namwong O, Apisariyakul A and Dunn FW: Inhibitory effect of dietary curcumin on skin carcinogenesis in mice. Cancer Lett 116: 197-203, 1997.

11. Srivastava R, Dikshit M, Srimal RC and Dhawan BN Anti-thrombotic effect of curcumin. Thromb Res 40: 413-417, 1985.

12. Nirmala $\mathrm{C}$ and Puvanakrishnan R: Protective role of curcumin against isoproterenol induced myocardial infarction in rats. Mol Cell Biochem 159: 85-93, 1996.

13. Nishiyama T, Mae T, Kishida H, Tsukagawa M, Mimaki Y, Kuroda M, Sashida Y, Takahashi K, Kawada T, Nakagawa K and Kitahara M: Curcuminoids and sesquiterpenoids in turmeric (Curcuma longa L.) suppress an increase in blood glucose level in type 2 diabetic KK-Ay mice. J Agric Food Chem 53: 959-963, 2005.

14. Funk JL, Oyarzo JN, Frye JB, Chen G, Lantz RC, Jolad SD, Sólyom AM and Timmermann BN: Turmeric extracts containing curcuminoids prevent experimental rheumatoid arthritis. J Nat Prod 69: 351-355, 2006.

15. Hong RL, Spohn WH and Hung MC: Curcumin inhibits tyrosine kinase activity of p185neu and also depletes p185neu. Clin Cancer Res 5: 1884-1891, 1999.

16. Huang MT, Smart RC, Wong CQ and Conney AH: Inhibitory effect of curcumin, chlorogenic acid, caffeic acid, and ferulic acid on tumor promotion in mouse skin by 12-O-tetradecanoylphorbol13-acetate. Cancer Res 48: 5941-5946, 1988.

17. Lin JK, Pan MH and Lin-Shiau SY: Recent studies on the biofunctions and biotransformations of curcumin. Biofactors 13 : $153-158,2000$

18. Choudhuri T, Pal S, Das T and Sa G: Curcumin selectively induces apoptosis in deregulated cyclin D1-expressed cells at G2 phase of cell cycle in a p53-dependent manner. J Biol Chem 280: 20059-20068, 2005
19. Ramachandran C and You W: Differential sensitivity of human mammary epithelial and breast carcinoma cell lines to curcumin. Breast Cancer Res Treat 54: 269-278, 1999.

20. Syng-Ai C, Kumari AL and Khar A: Effect of curcumin on normal and tumor cells: role of glutathione and bcl-2. Mol Cancer Ther 3: 1101-1108, 2004

21. Menon LG, Kuttan R and Kuttan G: Inhibition of lung metastasis in mice induced by B16F10 melanoma cells by polyphenolic compounds. Cancer Lett 95: 221-225, 1995.

22. Menon LG, Kuttan R and Kuttan G: Anti-metastatic activity of curcumin and catechin. Cancer Lett 141: 159-165, 1999.

23. Chen HW, Yu SL, Chen JJ, Li HN, Lin YC, Yao PL, Chou HY, Chien CT, Chen WJ, Lee YT and Yang PC: Anti-invasive gene expression profile of curcumin in lung adenocarcinoma based on a high throughput microarray analysis. Mol Pharmacol 65: 99-110, 2004.

24. Limtrakul P, Anuchapreeda S and Buddhasukh D: Modulation of human multidrug-resistance MDR-1 gene by natural curcuminoids. BMC Cancer 4: 13, 2004.

25. Mosmann T: Rapid colorimetric assay for cellular growth and survival: application to proliferation and cytotoxicity assays. J Immunol Methods 65: 55-63, 1983.

26. Savli H, Aalto Y, Nagy B, Knuutila S and Pakkala S: Gene expression analysis of 1,25(OH)2D3-dependent differentiation of HL-60 cells: a cDNA array study. Br J Haematol 118: 1065-1070, 2002.

27. Subramanian A, Tamayo P, Mootha VK, Mukherjee S, Ebert BL, Gillette MA, Paulovich A, Pomeroy SL, Golub TR, Lander ES and Mesirov JP: Gene set enrichment analysis: a knowledge-based approach for interpreting genome-wide expression profiles. Proc Natl Acad Sci USA 102: 15545-15550, 2005.

28. Aggarwal BB, Kumar A and Bharti AC: Anticancer potential of curcumin: preclinical and clinical studies. Anticancer Res 23: 363-398, 2003.

29. Salomon DS, Brandt R, Ciardiello F and Normanno N: Epidermal growth factor-related peptides and their receptors in human malignancies. Crit Rev Oncol Hematol 19: 183-232, 1995.

30. Arteaga CL: Epidermal growth factor receptor dependence in human tumors: more than just expression? Oncologist 7 (Suppl 4): 31-39, 2002.

31. Richards KN, Zweidler-McKay PA, Van Roy N, Speleman F, Trevino J, Zage PE and Hughes DP: Signaling of ERBB receptor tyrosine kinases promotes neuroblastoma growth in vitro and in vivo. Cancer 116: 3233-3243, 2010.

32. Hardy KM, Booth BW, Hendrix MJ, Salomon DS and Strizzi L: ErbB/EGF signaling and EMT in mammary development and breast cancer. J Mammary Gland Biol Neoplasia 15: 191-199, 2010.

33. Earp HS, Dawson TL, Li X and Yu H: Heterodimerization and functional interaction between EGF receptor family members: a new signaling paradigm with implications for breast cancer research. Breast Cancer Res Treat 35: 115-132, 1995.

34. Dancey JE and Freidlin B: Targeting epidermal growth factor receptor - are we missing the mark? Lancet 362: 62-64, 2003

35. Chen A, Xu J and Johnson AC: Curcumin inhibits human colon cancer cell growth by suppressing gene expression of epidermal growth factor receptor through reducing the activity of the transcription factor Egr-1. Oncogene 25: 278-287, 2006.

36. Shishodia S, Singh T and Chaturvedi MM: Modulation of transcription factors by curcumin. Adv Exp Med Biol 595: 127-148, 2007.

37. Choi BH, Kim CG, Bae YS, Lim Y, Lee YH and Shin SY: p21 Waf1/Cip1 expression by curcumin in U-87MG human glioma cells: role of early growth response- 1 expression. Cancer Res 68: 1369-1377, 2008

38. Chen A and Xu J: Activation of PPAR \{gamma\} by curcumin inhibits Moser cell growth and mediates suppression of gene expression of cyclin D1 and EGFR. Am J Physiol Gastrointest Liver Physiol 288: 447-456, 2005.

39. Han SS, Chung ST, Robertson DA, Ranjan D and Bondada S: Curcumin causes the growth arrest and apoptosis of B cell lymphoma by downregulation of egr-1, c-myc, bcl-XL, NF-kappa $\mathrm{B}$, and p53. Clin Immunol 93: 152-161, 1999.

40. Liu J, Liu YG, Huang R, Yao C, Li S, Yang W, Yang D and Huang RP: Concurrent down-regulation of Egr-1 and gelsolin in the majority of human breast cancer cells. Cancer Genomics Proteomics 4: 377-385, 2007.

41. Parra E and Ferreira J: The effect of siRNA-Egr-1 and camptothecin on growth and chemosensitivity of breast cancer cell lines. Oncol Rep 23: 1159-1165, 2010. 